\title{
RIGIDITY OF SURFACES WITH NO CONJUGATE POINTS
}

\author{
KEITH BURNS \& GERHARD KNIEPER
}

\begin{abstract}
E. Hopf proved that any complete Riemannian metric with no conjugate points on the torus $T^{2}$ is flat. We extend Hopf's argument to obtain sufficient conditions for metrics with no conjugate points on a cylinder or the plane to be flat.
\end{abstract}

\section{Introduction}

A complete Riemannian manifold has no conjugate points if any two points in its universal cover are joined by a unique geodesic. The no conjugate point property is a natural generalization of nonpositive curvature: any manifold with nonpositive curvature has no conjugate points by the Cartan-Hadamard theorem. In 1943 E. Hopf proved that a Riemannian metric with no conjugate points on the torus $T^{2}$ must be flat [8]. The present paper extends Hopf's arguments to obtain sufficient conditions for metrics on the cylinder $S^{1} \times \mathbb{R}$ and the plane $\mathbb{R}^{2}$ to be flat.

In the case of the cylinder, our main result-Theorem 2.2-is that a cylinder with no conjugate points and curvature bounded from below is flat if its ends do not open out, in other words if there is $L>0$ such that there is a nontrivial loop of length at most $L$ based at every point. This answers affirmatively a question raised in [6], where the result is proved under the stronger assumption that the cylinder has no focal points. Our method also shows that if the cylinder becomes thin as one approaches both ends, then there must be conjugate points. As a consequence, a cylinder with no conjugate points and curvature bounded from below has infinite area. We do not know whether the lower curvature bound can be removed in these results.

Received February 6, 1990 and, in revised form, June 21, 1990. The first author was supported by an Alfred P. Sloan Foundation Research Fellowship and by National Science Foundation Grant DMS 8896198. The second author was supported by the Sonderforschungsbereich 170, Geometrie und Analysis, Göttingen. 
In the case of the plane, we consider a version of Euclid's parallel axiom: we suppose that there is a constant $a \geq 1$ such that for every point $p$ and every geodesic $\gamma$ in $P$, there is a geodesic $\beta$ with $\beta(0)=p$ and

$$
\operatorname{dist}\left(\beta\left(t^{\prime}\right), \gamma\right) \leq a \operatorname{dist}(\beta(t), \gamma) \quad \text { for all } t, t^{\prime} \text {. }
$$

Our result-Theorem 3.1-is that any metric on the plane satisfying this axiom must be a flat Euclidean metric. An immediate corollary is the result of Green and Gulliver [7] that a flat metric on the plane cannot be changed on a compact set without introducing conjugate points. Other generalizations of Green and Gulliver's result have been obtained by Innami [9], [10] and Croke [3].

For surfaces with nonpositive curvature or no focal points all of the above results are simple corollaries of the following result.

0.1. Flat Strip Theorem [4], [12], [5]. Let $\beta$ and $\gamma$ be geodesics in a simply connected manifold with nonpositive curvature or no focal points. Suppose that $\beta$ and $\gamma$ have finite Hausdorff distance. Then $\beta$ and $\gamma$ are the edges of a flat strip, i.e., an isometrically and totally geodesically embedded copy of $I \times \mathbb{R}$.

The obvious generalization of this theorem to manifolds with no conjugate points is false; a compact two dimensional counterexample is constructed in [2]. This example does not, however, contradict the following conjecture.

0.2. Conjecture. Let $S$ be a simply connected surface with a complete Riemannian metric with no conjugate points. Suppose that $S$ is foliated by a family of geodesics, any two of which have finite Hausdorff distance. Then $S$ is flat.

All of the results of the present paper would follow easily if this conjecture were true.

Work on this paper began while both authors were visiting the University of North Carolina as part of a special year in differential geometry sponsored by the University and the National Science Foundation. We thank the University and the organizers, Pat Eberlein and Robbie Gardner, for their hospitality. We also thank Chris Croke for the discussion which led to Theorem 2.8 , and the referee for a very careful reading of the paper.

\section{Preliminaries}

Throughout this paper $S$ will be a smooth surface with a complete Riemannian metric. We shall always measure angles so that they take 
values in $[0, \pi]$. Let $K$ be the Gaussian curvature and $\pi$ the projection from $T S$ to $S$. If $X \subseteq S, T^{1} X$ will denote the set of unit vectors with footpoint in $X$ and $\partial^{1} X$ the set of all unit vectors with footpoint in $\partial X$. Unless otherwise mentioned, geodesics have unit speed. If $v \in T^{1} S, \gamma_{v}$ is the geodesic with $\dot{\gamma}_{v}(0)=v$. The geodesic flow $g^{t}$ on $T^{1} S$ is defined by $g^{t}(v)=\dot{\gamma}_{v}(t)$. Let $\nu$ be the area defined on $S$ by the Riemannian metric and $\mu$ the Liouville measure on $T^{1} S$. Also $\lambda$ will denote the measure that is the product of Lebesgue measure on the fibers of $T^{1} S$ with the Riemannian length measure on a rectifiable one-dimensional subset of $S$; it will always be clear from the context which one-dimensional set is intended.

We consider the scalar Jacobi equation along the geodesic $\gamma_{v}$ :

$$
y^{\prime \prime}(t)+K\left(\gamma_{v}(t)\right) y(t)=0 .
$$

If $N(t)$ is a continuous vector field normal to $\gamma_{v}$, then $y(t)$ is a solution to (1.1) if and only if $y(t) N(t)$ is a Jacobi field along $\gamma_{v}$.

1.1. Definition. Two points $\gamma_{v}\left(t_{1}\right)$ and $\gamma_{v}\left(t_{2}\right)$ are conjugate along $\gamma_{v}$ if there is a solution $y(t)$ of (1.1) that has $y\left(t_{1}\right)=0=y\left(t_{2}\right)$ and does not vanish identically. The surface $S$ has no conjugate points if no pair of points is conjugate along any geodesic.

It is well known that this definition is equivalent to the characterization of surfaces with no conjugate points given earlier, that $S$ has no conjugate points if and only if any two points $p$ and $q$ in the universal cover $\widetilde{S}$ are joined by a unique geodesic $\gamma_{p, q}$ with $\gamma_{p, q}(0)=p$ and $\gamma_{p, q}(\operatorname{dist}(p, q))=$ $q$. In particular, if $S$ has no conjugate points, $\exp _{p}: T_{p} \widetilde{S} \rightarrow \widetilde{S}$ is a diffeomorphism for every $p \in \tilde{S}$. Thus a simply connected surface with no conjugate points is diffeomorphic to $\mathbb{R}^{2}$.

Let $z(v, t)$ be the solution of (1.1) with $z(v, t)=0$ and $z^{\prime}(v, t)=1$. Then $S$ has no conjugate points if and only if, for every $v, z(v, t) \neq 0$ when $t \neq 0$. If there are no conjugate points along $\gamma_{v}$, there is for each $s \neq 0$ a well-defined solution $y(v, s, t)$ of (1.1) with $y(v, s, 0)=1$ and $y(v, s, s)=0$. Moreover

$$
y_{-}(v, t)=\lim _{s \rightarrow \infty} y(v, s, t) \quad \text { and } \quad y_{+}(v, t)=\lim _{s \rightarrow-\infty} y(v, s, t)
$$

are well-defined solutions of $(1.1)$ with $y_{-}^{\prime}(0) \leq y_{+}^{\prime}(0)$; see e.g. [8]. A solution $y$ of (1.1) with $y(0)=1$ has $y(t)>0$ for all $t \leq 0$ if and only if $y^{\prime}(0) \leq y_{+}^{\prime}(0)$, and has $y(t)>0$ for all $t \geq 0$ if and only if $y^{\prime}(0) \geq y_{-}^{\prime}(0)$. We call $y_{-}$and $y_{+}$the stable and unstable solutions respectively of (1.1). 
Solving (1.1) by reduction of order shows that if $\gamma_{v}$ has no conjugate points, then

$$
z(v, t)=y_{+}(v, t) \int_{0}^{t} \frac{d t^{\prime}}{y_{+}^{2}\left(v, t^{\prime}\right)}
$$

The Riccati equation

$$
u^{\prime}(t)+u^{2}(t)+K\left(\gamma_{v}\right)=0
$$

is obtained from (1.1) by the change of variable $u=y^{\prime} / y$. The times $t_{1}<t_{2}$ are consecutive zeroes of a solution $y$ of (1.1) if and only if the corresponding solution $u$ of $(1.3)$ is defined throughout $\left(t_{1}, t_{2}\right)$ and $u(t) \rightarrow \infty$ as $t \searrow t_{1}$ and $u(t) \rightarrow-\infty$ as $t \nearrow t_{2}$. Thus there are no conjugate points along $\gamma_{v}$ if and only if (1.3) has a solution that is defined for all $t$. If $\gamma_{v}$ has no conjugate points, we set $u_{+}(t)=y_{+}^{\prime} / y_{+}(t)$ and $u_{-}(t)=y_{-}^{\prime} / y_{-}(t)$. It is clear that $u_{+}$and $u_{-}$are the largest and smallest solutions respectively of (1.3) that are defined for all $t$.

1.2. Proposition. Suppose that the surface $S$ has no conjugate points. Then the following hold.

(i) $u_{ \pm}(\cdot, \cdot)$ are measurable functions.

(ii) $u_{ \pm}\left(g^{t} v, s\right)=u_{ \pm}(v, s+t)$ for all $s$ and $t$.

(iii) If in addition $K(p) \geq-b^{2}$ for all $p \in S$, then $\left|u_{ \pm}(v, t)\right| \leq b$ for all $(v, t)$. In particular, if $u(t)$ is a solution of (1.3) with $u(0)>b$ (resp. $u(0)<-b)$, then the corresponding solution $y(t)$ of (1.1) vanishes for some $t<0$ (resp. some $t>0)$.

Proof. See [8] or [1].

We set $U=u_{+}(\cdot, 0)$. Our arguments are based on

1.3. Key Lemma. Suppose that $Q$ is a compact subset of $S$ whose boundary is a piecewise smooth curve. Then

$$
\int_{T^{1} Q} U^{2}(v) d \mu(v) \leq-2 \pi \int_{Q} K(p) d \nu(p)+2 \int_{\partial^{1} Q}|U(v)| d \lambda .
$$

Proof. Integrating the Riccati equation (1.3) shows that for any $\tau>0$,

$$
\int_{T^{1} Q} \frac{1}{\tau} \int_{0}^{\tau} u_{+}^{\prime}\left(g^{t} v, 0\right)+u_{+}^{2}\left(g^{t} v, 0\right)+K\left(\gamma_{v}(t)\right) d t d \mu(v)=0 .
$$


Since the Liouville measure $\mu$ is $g^{t}$-invariant, letting $\tau \rightarrow 0$ gives

$$
\begin{aligned}
\int_{T^{1} Q} U^{2}(v) d \mu(v) \leq & -\int_{T^{1} Q} K(\pi v) d \mu(v) \\
& +\limsup _{\tau \rightarrow 0}\left|\int_{T^{1} Q} \frac{1}{\tau} \int_{0}^{\tau} u_{+}^{\prime}\left(g^{t} v, 0\right) d t d \mu\right| .
\end{aligned}
$$

Of course

$$
-\int_{T^{1} Q} K(\pi v) d \mu(v)=-2 \pi \int_{Q} K(p) d \nu(p) .
$$

It follows from the invariance property (Proposition 1.2(ii)) of $u_{+}$and the $g^{t}$-invariance of $\mu$ that

$$
\begin{aligned}
\int_{T^{1} Q} \int_{0}^{\tau} u_{+}^{\prime}\left(g^{t} v, 0\right) d t d \mu & =\int_{T^{1} Q}\left\{U\left(g^{\tau} v\right)-U(v)\right\} d \mu(v) \\
& =\int_{g^{\tau}\left(T^{1} Q\right)} U(v) d \mu(v)-\int_{T^{1} Q} U(v) d \mu(v) \\
& =\int_{g^{\tau}\left(T^{1} Q\right) \Delta T^{1} Q} U(v) d \mu(v) .
\end{aligned}
$$

Observe that $g^{\tau}\left(T^{1} Q\right) \Delta T^{1} Q \subseteq G^{\tau}\left(\partial^{1} Q\right) \stackrel{\text { def }}{=}\left\{g^{t}(v): v \in \partial^{1} Q\right.$ and $|t| \leq$ $\tau\}$. Define $\varphi: \partial^{1} Q \times \mathbb{R} \rightarrow T^{1} S$ by $\varphi(v, t)=g^{t} v$. Then

$$
\begin{aligned}
\left|\int_{T^{1} Q} \int_{0}^{\tau} u_{+}^{\prime}\left(g^{t} v, 0\right) d t d \mu\right| & \leq \int_{G^{\tau}\left(\partial^{1} Q\right)}|U(v)| d \mu(v) \\
& \leq D(\tau) \int_{\partial^{1} Q} \int_{-\tau}^{\tau}\left|U\left(g^{t} v\right)\right| d t d \lambda(v),
\end{aligned}
$$

where

$$
D(\tau)=\sup \left\{|\operatorname{det} D \varphi(v, t)|:(v, t) \in \partial^{1} Q \times[-\tau,-\tau]\right.
$$

and $\partial Q$ is smooth at $\pi v\}$.

Since $g^{t}$ is a unit speed flow, it is easily shown that $\lim \sup _{\tau \rightarrow 0} D(\tau) \leq 1$. 
It follows that

$$
\begin{aligned}
\limsup _{\tau \rightarrow 0} \mid & \int_{T^{1} Q} \frac{1}{\tau} \int_{0}^{\tau} u_{+}^{\prime}\left(g^{t} v, 0\right) d t d \mu(v) \mid \\
\leq & \int_{\partial^{1} Q} \limsup _{\tau \rightarrow 0} \frac{1}{\tau} \int_{-\tau}^{\tau}\left|U\left(g^{t}\right)\right| d t d \mu(v) \leq 2 \int_{\partial^{1} Q}|U(v)| d \lambda,
\end{aligned}
$$

since $U\left(g^{t} v\right)=u_{+}(v, t)$ is a continuous (actually $\left.C^{\infty}\right)$ function of $t$ for each fixed $v$. q.e.d.

When we have the lower curvature bound $K \geq-b^{2}$, we shall often compare solutions of (1.1) with solutions of the scalar Jacobi equation in constant curvature $-b^{2}$ using of

1.4. Lemma. Suppose $K_{1}(t) \geq K_{2}(t)$ for all $t$ and $y_{i}(t)$ is a solution

$$
y_{i}^{\prime \prime}(t)+K_{i}(t) y_{i}(t)=0, \quad i=1,2 .
$$

If $0 \leq y_{1}(0)=y_{2}(0), 0 \leq y_{1}(0) \leq y_{2}(0)$ and $y_{1}(t) \geq 0$ for $0 \leq t \leq t_{0}$, then $y_{1}\left(t_{0}\right) \leq y_{2}\left(t_{0}\right)$.

In particular, if $K\left(\gamma_{v}(t)\right) \geq-b^{2}$ for all $t$ and there are no conjugate points along $\gamma_{v}$, then

$$
z(v, t) \leq \frac{1}{b} \sinh (b t), \quad t \geq 0 .
$$

We also use two fundamental results of Leon Green; they are Theorems 2.1 and 3.1 of [6].

1.5. Lemma. Suppose there are no conjugate points along $\gamma_{v}$ and $K\left(\gamma_{v}(t)\right)$ is bounded from below. Then $z(v, t) \rightarrow \infty$ as $t \rightarrow \infty$.

1.6. Proposition. Let $S$ be a complete simply connected surface with no conjugate points and curvature bounded from below. Let $\gamma$ be a geodesic in $S$. Suppose $\left\{p_{n}\right\}$ is a sequence such that $\operatorname{dist}\left(p_{n}, \gamma(0)\right) \rightarrow \infty$ and $\operatorname{dist}\left(p_{n}, \gamma\right)$ is bounded. Then

$$
\dot{\gamma}_{\gamma(0), p_{n}}(0) \rightarrow \dot{\gamma}(0) \text {. }
$$

In particular if $\beta$ and $\gamma$ are two geodesics with $\beta(0)=\gamma(0)$ and $\dot{\beta}(0) \neq$ $\dot{\gamma}(0)$, then $\operatorname{dist}(\beta(t), \gamma(t)) \rightarrow \infty$ as $t \rightarrow \infty$.

If $S$ is simply connected and has no conjugate points, we can consider the following notions of parallelism and asymptoticity for two geodesics $\beta$ and $\gamma$ in $S$ :

(i) $\operatorname{dist}(\beta(t), \gamma(t))$ is constant; 
(ii) there is $b \geq 1$ such that $\operatorname{dist}\left(\beta\left(t^{\prime}\right), \gamma\left(t^{\prime}\right)\right) \leq b \operatorname{dist}(\beta(t), \gamma(t))$ for all $t$ and $t^{\prime}$;

(iii) $\sup \{\operatorname{dist}(\beta(t), \gamma(t)): t \in \mathbb{R}\}<\infty$;

(iv) $\beta$ and $\gamma$ have finite Hausdorff distance;

(v) $\beta$ and $\gamma$ do not intersect;

(vi) $\beta$ is asymptotic to $\gamma$, i.e., $\dot{\beta}(0)=\lim _{t \rightarrow \infty} \dot{\gamma}_{\beta(0), \gamma(t)}(0)$.

Note that for every geodesic $\gamma$ of $S$, there is exactly one geodesic asymptotic to $\gamma$ starting from each point of $S$ [5, Proposition 1]. Our definition of asymptoticity follows [5] and is not the definition usually used in the theory of manifolds with nonpositive curvature, namely that $\beta$ and $\gamma$ are asymptotic if $\sup \{\operatorname{dist}(\beta(t), \gamma(t)): t \geq 0\}<\infty$. The two definitions are equivalent in the context of manifolds with nonpositive curvature or with no focal points. For such manifolds, the flat strip theorem shows that (i) $\Leftrightarrow$ (ii) $\Leftrightarrow$ (iii). It is not difficult to see that (iii) $\Leftrightarrow$ (iv) in any simply connected manifold with no conjugate points, and it is trivial that (i) $\Rightarrow$ (ii) $\Rightarrow$ (iii). For surfaces with no conjugate points and curvature bounded from below, Proposition 1.6 shows that (iii) $\Rightarrow$ (iv), (v) and that $\gamma$ is asymptotic to $\beta$. However neither (ii) nor (iii) implies (i), even if the curvature is bounded from below [2].

Property (vi) characterizes surfaces with no conjugate points in the following way.

1.7. Proposition. Assume that $S$ is simply connected. Then $S$ has no conjugate points if and only if, for any geodesic $\gamma$ and any point $p$ not on $\gamma$, there is a geodesic $\beta$ with $\beta(0)=p$ that does not intersect $\gamma$.

Proof. Suppose that $S$ has no conjugate points. Let $v^{ \pm}$be the vectors in $T_{p}^{1} S$ such that $\gamma_{v^{+}}$is asymptotic to $\gamma$ and $\gamma_{v^{-}}$is asymptotic to the geodesic $t \mapsto \gamma(-t)$. Let $v$ be the unit vector pointing from $p$ towards $\gamma(0)$. Let $A$ be the connected open arc in the circle $T_{p}^{1} S$ that contains $v$ and is bounded by $v^{-}$and $v^{+}$. If $u \in T_{p}^{1} S$, the ray $\gamma_{u} \mid[0, \infty)$ intersects $\gamma$ if and only if $u \in A$. Note that $-u \notin A$ if $u \in A$, for otherwise $\gamma_{u}$ would intersect $\gamma$ twice. Thus the open arc $A$ lies in the interior of a semicircle in $T_{p}^{1} S$, and we can find $w \in T_{p}^{1} S$ with $w \notin A$ and $-w \notin A$. The geodesic $\beta=\gamma_{w}$ does not intersect $\gamma$.

Conversely suppose that $S$ has conjugate points. Choose a point $p \in S$ that lies between a pair of conjugate points along some geodesic. Let $\rho=$ $\inf \{r: \delta(-r)$ and $\delta(r)$ are conjugate along a geodesic $\delta$ with $\delta(0)=p\}$. Note that, for every $u \in T_{p}^{1} S$, we have $z(u, t)>0$ for $0<t \leq \rho$. Since $z$ is continuous and $T_{p}^{1} S$ is compact, there is $R>\rho$ such that 
$z(u, t)>0$ for all $(u, t) \in T_{p}^{1} S \times(0, R]$. Hence $\exp _{p}$ is nonsingular and injective on the ball $B=\left\{v \in T_{p} S:\|v\|<R\right\}$. Let $g$ be the metric on $B$ that is the pullback by $\exp _{p}$ of the metric on $S$. Choose a geodesic $\delta_{0}$ in $S$ with $\delta_{0}(0)=p$ and $\delta_{0}(-\rho)$ conjugate to $\delta_{0}(\rho)$ along $\delta_{0}$. Then $\tilde{\delta}_{0}(t) \stackrel{\text { def }}{=} t \dot{\delta}_{0}(0)$ is a geodesic of $(B, g)$. Since $\tilde{\delta}_{0}(-\rho)$ and $\tilde{\delta}_{0}(\rho)$ are conjugate along $\tilde{\delta}_{0}$, a geodesic of $(B, g)$ that passes through $\tilde{\delta}_{0}(-\rho)$ and makes a small (but nonzero) angle with $\tilde{\delta}_{0}$ will intersect $\tilde{\delta}_{0}$ near $\tilde{\delta}_{0}(\rho)$; see $[11,2.1 .13]$. Such a geodesic cannot pass through 0 , since this would contradict injectivity of $\exp _{p}$ on $B$. Thus there is a geodesic segment $\tilde{c}$ in $(B, g)$ that joins $\tilde{\delta}_{0}(-\rho)$ to $\tilde{\delta}_{0}(\tau)$ for some $\tau \in(0, R)$ and does not pass through 0 . Every geodesic in $(B, g)$ that passes through 0 crosses $\tilde{c}$. Hence every geodesic in $S$ that passes through $p$ must cross the geodesic segment $c=\exp _{p} \circ \tilde{c}$. The extension $\gamma$ of $c$ to a complete geodesic may contain $p$. Choose a point $p^{\prime}$ that lies on $\delta_{0}$ between the endpoints of $c$ and does not lie on $\gamma$. We can choose $p^{\prime}$ so close to $p$ that $\exp _{p^{\prime}}$ is nonsingular on $B^{\prime}=\left\{v \in T_{p^{\prime}} S:\|v\|<R\right\}$ and $c$ lies in $\exp _{p^{\prime}} B^{\prime}$. Then every geodesic that passes through $p^{\prime}$ intersects $\gamma$.

\section{Cylinders with bounded cross section}

Let $C$ be a cylinder $\left(S^{1} \times \mathbb{R}\right)$ with a complete Riemannian metric. Let $\varphi$ be a generator of $\pi_{1}(C)$ thought of as the group of covering transformations acting as isometries on the Riemannian universal cover $\widetilde{C}$ of C.

2.1. Definition. $C$ has bounded cross section if there is $L$ such that $\operatorname{dist}(p, \varphi p) \leq L$ for all $p \in \widetilde{C}$.

An equivalent statement is that there be a non-null-homotopic loop with length $\leq L$ based at each point of $C$.

2.2. Theorem. Suppose $C$ has no conjugate points, curvature bounded from below and bounded cross section. Then $C$ is flat.

Proof. Choose $b, L>0$ so that $K(p) \geq-b^{2}$ and $\operatorname{dist}(p, \varphi p) \leq L$ for all $p \in \widetilde{C}$. We shall show below that the function $U=u_{+}(\cdot, 0)$ is in $L^{2}\left(T^{1} C\right)$ and satisfies $\int_{T^{1} C} U^{2}(v) d \mu(v)=0$. This implies that $U$ vanishes almost everywhere. It then follows from the Riccati equation (1.3) that the curvature $K$ vanishes almost everywhere. Since $K$ is continuous, $C$ must be flat.

We first construct a geodesic $\gamma_{0}$ that has no self-intersections and joins the two ends of $C$. To do this, choose an increasing sequence $\left\{K_{n}\right\}$ 
of compact subsets of $C$ such that $C=\bigcup_{n=1}^{\infty} K_{n}$ and each $C \backslash K_{n}$ has precisely two components $A_{n}$ and $B_{n}$. Let $g_{n}$ be the shortest geodesic segment joining a point in $A_{n}$ to a point in $B_{n}$. Let $\gamma_{0}$ be a geodesic that is a limit of $\left\{g_{n}\right\}$. Then $\gamma_{0}$ is minimal and in particular has no selfintersections. For any $n \geq m, g_{n}$ contains points of both the compact subsets $\partial A_{m}$ and $\partial B_{m}$. Hence $\gamma_{0}$ contains points of both $\partial A_{m}$ and $\partial B_{m}$ for every $m$. Since $\gamma_{0}$ is minimal, it follows easily that $\gamma_{0}(s)$ approaches one end of $C$ as $s \rightarrow \infty$ and the other end as $s \rightarrow-\infty$.

Choose a lift $\gamma_{1}$ of $\gamma_{0}$ to $\widetilde{C}$. Let $\gamma_{2}=\varphi \circ \gamma_{1}$. Since $\gamma_{0}$ has no selfintersections, $\gamma_{1}$ and $\gamma_{2}$ do not intersect. They bound a strip $\Sigma$ that is a fundamental domain for $\pi_{1}(C)$.

Let $l(s)=\operatorname{dist}\left(\gamma_{1}(s), \gamma_{2}(s)\right)$. Note that $l(s) \leq L$ for all $s$. Let $\sigma_{s}$ be the geodesic of $\widetilde{C}$ with $\sigma_{s}(0)=\gamma_{1}(s)$ and $\sigma_{s}(l(s))=\gamma_{2}(s)$. We shall call the segment of $\sigma_{s}$ between $\gamma_{1}(s)$ and $\gamma_{2}(s)$ the cross section of $\Sigma$ at $s$. Two different cross sections of $\Sigma$ cannot intersect: if $s \neq s^{\prime}$, the points $\gamma_{1}\left(s^{\prime}\right)$ and $\gamma_{2}\left(s^{\prime}\right)$ lie on the same side of $\sigma_{s}$, and so the geodesic segment joining them cannot intersect $\sigma_{s}$. Let $c_{s}$ be the projection to $C$ of the cross section of $\Sigma$ at $s$ and let $T^{1}(s)$ be the set of all unit vectors based at points on $c_{s}$. Note that

$$
\lambda\left(T^{1}(s)\right) \leq 2 \pi L \quad \text { for all } s .
$$

Let $\alpha(s)=\varangle\left(\dot{\sigma}_{s}(0), \dot{\gamma}_{1}(s)\right)$ and $\beta(s)=\varangle\left(-\dot{\sigma}_{s}(l(s)), \dot{\gamma}_{2}(s)\right)$. Let $\hat{\alpha}(s)=$ $\varangle\left(\dot{\sigma}_{s}(0),-\dot{\gamma}_{1}(s)\right)=\pi-\alpha(s)$ and $\hat{\beta}(s)=\varangle\left(-\dot{\sigma}_{s}(l(s)),-\dot{\gamma}_{2}(s)\right)=\pi-\beta(s)$. Assume that $s^{\prime}<s^{\prime \prime}$ and consider the set $Q\left(s^{\prime}, s^{\prime \prime}\right) \subseteq C$ consisting of points that lie on or between $c_{s^{\prime}}$ and $c_{s^{\prime \prime}}$. The lift of $Q\left(s^{\prime}, s^{\prime \prime}\right)$ to $\Sigma$ is a quadrilateral with geodesic sides, whose interior angles are $\hat{\alpha}\left(s^{\prime \prime}\right), \hat{\beta}\left(s^{\prime \prime}\right)$, $\alpha\left(s^{\prime}\right)$ and $\beta\left(s^{\prime}\right)$. It follows from the Gauss-Bonnet theorem that

$$
-\int_{Q\left(s^{\prime}, s^{\prime \prime}\right)} K(p) d \nu(p)=\left\{2 \pi-\hat{\alpha}\left(s^{\prime \prime}\right)-\hat{\beta}\left(s^{\prime \prime}\right)-\alpha\left(s^{\prime}\right)-\beta\left(s^{\prime}\right)\right\},
$$

which together with Lemma 1.3 implies that

$$
\begin{gathered}
\int_{T^{1} Q\left(s^{\prime}, s^{\prime \prime}\right)} U^{2}(v) d \mu(v) \leq 2 \pi\left\{2 \pi-\hat{\alpha}\left(s^{\prime \prime}\right)-\hat{\beta}\left(s^{\prime \prime}\right)-\alpha\left(s^{\prime}\right)-\beta\left(s^{\prime}\right)\right\} \\
+2 \int_{T^{1}\left(s^{\prime}\right) \cup T^{1}\left(s^{\prime \prime}\right)}|U(v)| d \lambda(v) .
\end{gathered}
$$

Note that $-2 \pi \leq\left\{2 \pi-\hat{\alpha}\left(s^{\prime \prime}\right)-\hat{\beta}\left(s^{\prime \prime}\right)-\alpha\left(s^{\prime}\right)-\beta\left(s^{\prime}\right)\right\} \leq 2 \pi$ and $|U(v)| \leq b$ 
for all $v$ by (iii) of Proposition 1.2. It follows from this and (2.1) that

$$
\int_{Q\left(s^{\prime}, s^{\prime \prime}\right)} U^{2}(v) d \mu(v) \leq 4 \pi^{2}+4 \pi b L \quad \text { whenever } s^{\prime}<s^{\prime \prime} .
$$

Thus $U \in L^{2}\left(T^{1} C\right)$.

2.3. Lemma. There is $\Theta>0$ such that every cross section of $\Sigma$ makes angle at least $\Theta$ with $\gamma_{1}$ and $\gamma_{2}$.

Proof. In fact one can take

$$
\Theta=\frac{b L}{3 \sinh b L} .
$$

We give the proof for the angle with $\gamma_{1}$. For a given $s$, let $\theta$ be the angle between $\sigma_{s}$ and $\gamma_{1}$, and set $l=l(s)$. We have $\operatorname{dist}\left(\sigma_{s}(l), \gamma_{1}\right) \leq$ $\operatorname{dist}\left(\sigma_{s}(l), \gamma_{1}(s+l)\right)$, which in turn is less than the distance between these points along the circle with radius $l$ centered at $\sigma_{s}(0)$. Comparison with an arc subtending angle $\theta$ at the center of a circle of radius $l$ in constant curvature $-b^{2}$ (using (1.5)) shows that

$$
\operatorname{dist}\left(\sigma_{s}(l), \gamma_{1}\right) \leq \frac{\theta}{b} \sinh b l=l \theta \frac{\sinh b l}{b l} \leq l \theta \frac{\sinh b L}{b L},
$$

since $l \leq L$ and $x^{-1} \sinh x$ is an increasing function. Thus if $\theta \leq \Theta$, we have

$$
\operatorname{dist}\left(\gamma_{2}(s), \gamma_{1}\right)=\operatorname{dist}\left(\sigma_{s}(l), \gamma_{1}\right) \leq l / 3
$$

Choose $s^{\prime}$ so that $\operatorname{dist}\left(\gamma_{2}(s), \gamma_{1}\left(s^{\prime}\right)\right) \leq l / 3$. Projecting from $\tilde{C}$ to $C$ shows that $\gamma_{0}(s)$ and $\gamma_{0}\left(s^{\prime}\right)$ are joined by a curve of length $\leq l / 3$. Since $\gamma_{0}$ is a minimal geodesic, $\left|s-s^{\prime}\right| \leq l / 3$. Thus

$$
l=\operatorname{dist}\left(\gamma_{1}(s), \gamma_{2}(s)\right) \leq \operatorname{dist}\left(\gamma_{1}(s), \gamma_{1}\left(s^{\prime}\right)\right)+\operatorname{dist}\left(\gamma_{1}\left(s^{\prime}\right), \gamma_{2}(s)\right) \leq \frac{2 l}{3},
$$

which is impossible. Hence $\theta \geq \Theta$.

2.4. Lemma. There are $m, M>0$ such that, if $s^{\prime}<s^{\prime \prime}$ and $f$ : $T^{1} Q\left(s^{\prime}, s^{\prime \prime}\right) \rightarrow[0, \infty)$ is integrable, then

$$
\begin{aligned}
m \int_{s^{\prime}}^{s^{\prime \prime}} \int_{T^{1}(s)} f(v) d \lambda(v) d s & \leq \int_{T^{1} Q\left(s^{-}, s^{+}\right)} f(v) d \mu(v) \\
& \leq M \int_{s^{\prime}}^{s^{\prime \prime}} \int_{T^{1}(s)} f(v) d \lambda(v) d s .
\end{aligned}
$$

Proof. Set $\psi(s, t)=\sigma_{s}(t)$, and let $y_{s}(t)$ be the length the projection of $(\partial \psi / \partial s)(s, t)$ onto the direction orthogonal to $\sigma_{s}$. Then $y_{s}$ is a scalar 
Jacobi field along $\sigma_{s}$. Since two different cross sections of $\Sigma$ cannot intersect, $y_{s}(t)>0$ for $0<t<l(s)$.

To prove the lemma, it suffices to choose $m$ and $M$ so that $m \leq$ $y_{s}(t) \leq M$ whenever $0 \leq t \leq l(s)$. Since $\dot{\gamma}_{1}(s)=(\partial \psi / \partial s)(s, 0)$ and

$$
\dot{\gamma}_{2}(s)=\frac{d}{d s}\{\psi(s, l(s))\}=\frac{\partial \psi}{\partial s}(s, l(s))+l^{\prime}(s) \dot{\sigma}_{s}(l(s)),
$$

$y_{s}(0)$ and $y_{s}(l(s))$ are the components orthogonal to $\sigma_{s}$ of $\dot{\gamma}_{1}(s)$ and $\dot{\gamma}_{2}(s)$ respectively. It follows from Lemma 2.3 that

$$
\sin \Theta \leq y_{s}(0), y_{s}(l(s)) \leq 1 \quad \text { for all } s .
$$

Let

$$
m=\frac{\sin \Theta}{\cosh b L} .
$$

Suppose that for some $s$ we have $y_{s}(t)<m$ for $t \in[0, l(s)]$. Since $m<\sin \Theta$, the function $y_{s} \mid[0, l(s)]$ must attain its infimum at a time $t_{0} \in(0, l(s))$ where its derivative vanishes. Comparison with constant curvature $-b^{2}$ gives $y_{s}(0) \leq y_{s}\left(t_{0}\right) \cosh b t_{0}<m \cosh b L<\sin \Theta$, which is impossible. Thus $y_{s}(t)>m$ whenever $0 \leq t \leq l(s)$.

Let $u_{s}(t)=y_{s}^{\prime}(t) y_{s}^{-1}(t)$. If $u_{s}(0)>b$, then $y_{s}(t)$ vanishes for some $t<0$ by (iii) of Proposition 1.2. Similarly if $u_{s}(l(s))<-b$, then $y_{s}(t)$ vanishes for some $t>l(s)$. But $y_{s}(t)$ vanishes at most once. From this and (2.3) we see that

(i) $y_{s}^{\prime}(0) \leq b y_{s}(0) \leq b$, or

(ii) $y_{s}^{\prime}(l(s)) \geq-b y_{s}(l(s)) \geq-b$.

Suppose (i) holds. Comparing with constant curvature $-b^{2}$ and using Lemma 1.4 show that $y_{s}(t) \leq e^{b t}$ for $t \geq 0$, since $e^{b t}$ is the solution of the initial value problem $y^{\prime \prime}(t)-b^{2} y(t)=0, y(0)=1, y^{\prime}(0)=b$. Hence

$$
y_{s}(t) \leq e^{b t} \leq e^{b l(s)} \leq e^{b L},
$$

for $0 \leq t \leq l(s)$. A similar argument leads to that if (ii) holds and $0 \leq t \leq l(s)$, then

$$
y_{s}(l(s)-t) \leq e^{b t} \leq e^{b L} .
$$

Thus we can take $M=e^{b L}$. q.e.d.

We are now ready to prove that $\int_{T^{1} C} U^{2} d \mu(v)=0$. In fact we shall show that, if $s_{0}>0$ and $0<\varepsilon<10 \pi$, there are $s^{-}<-s_{0}$ and $s^{+}>s_{0}$ such that

$$
\int_{T^{1} Q\left(s^{-}, s^{+}\right)} U^{2}(v) d \mu(v) \leq \varepsilon .
$$


Choose $s_{1}>s_{0}$ large enough so that $s_{1}>1$,

$$
\int_{T^{1} Q\left(-s_{1}, s_{1}\right)} U^{2}(v) d \mu(v)>\int_{T^{1} C} U^{2}(v) d \mu(v)-\frac{m^{2} \varepsilon^{2}}{1600 \pi L M},
$$

and

$$
\frac{\varepsilon^{2}}{50 \pi^{4}}\left(s_{1}-\sqrt{s_{1}}\right)-2 \sqrt{s_{1}}>L .
$$

It is convenient to say that $s$ is good if

$$
\int_{T^{1}(s)}|U(v)| d \lambda(v) \leq \frac{\varepsilon}{20} .
$$

We choose $s^{+} \in\left[s_{1}, 2 s_{1}\right]$ and $s^{-} \in\left[-2 s_{1},-s_{1}\right]$ so that they are good. Since $s_{1}>1$, the next lemma shows that this is possible.

2.5. Lemma. Both of the sets $\left\{s \in\left[s_{1}, 3 s_{1}\right]: s\right.$ is not good $\}$ and $\left\{s \in\left[-3 s_{1},-s_{1}\right]: s\right.$ is not good $\}$ have length at most $\sqrt{s_{1}}$.

Proof. We consider the first set; the other case is similar.

$$
\begin{aligned}
m \cdot \frac{\varepsilon}{20} & \text { length }\left\{s \in\left[s_{1}, 3 s_{1}\right]: s \text { is not good }\right\} \\
& \leq m \int_{s_{1}}^{3 s_{1}} \int_{T^{1}(s)}|U(v)| d \lambda(v) d s \\
& \leq \int_{T^{1} Q\left(s_{1}, 3 s_{1}\right)}|U(v)| d \mu(v) \text { by Lemma } 2.4 \\
& \leq\left\{\int_{T^{1} Q\left(s_{1}, 3 s_{1}\right)} U^{2}(v) d \mu(v)\right\}^{1 / 2}\left\{\int_{T^{1} Q\left(s_{1}, 3 s_{1}\right)}^{1 d \mu(v)}\right\}^{1 / 2} \\
& \leq \frac{m \varepsilon}{40 \sqrt{\pi L M}}\left\{M \int_{s_{1}}^{3 s_{1}} \int_{T^{1}(s)}^{1 / 2} 1 d \lambda(v) d s\right\}^{\text {by }(2.5) \text { and }} \text { Lemma } 2.4 \\
& \leq \frac{m \varepsilon}{40 \sqrt{\pi L M}} \sqrt{4 \pi L M s_{1}} \text { by }(2.1) \\
& =m \cdot \frac{\varepsilon}{20} \cdot \sqrt{s_{1}} . \quad \text { q.e.d. }
\end{aligned}
$$


Since $s^{+}$and $s^{-}$are good, (2.2) gives

$$
\int_{T^{1} Q\left(s^{-}, s^{+}\right)} U^{2}(v) d \mu(v) \leq 2 \pi\left\{2 \pi-\hat{\alpha}\left(s^{+}\right)-\hat{\beta}\left(s^{+}\right)-\alpha\left(s^{-}\right)-\beta\left(s^{-}\right)\right\}+\frac{\varepsilon}{5} \text {. }
$$

Thus (2.4) will hold if

$$
\pi-\hat{\alpha}\left(s^{+}\right)-\hat{\beta}\left(s^{+}\right) \leq \frac{\varepsilon}{5 \pi} \quad \text { and } \quad \pi-\alpha\left(s^{-}\right)-\beta\left(s^{-}\right) \leq \frac{\varepsilon}{5 \pi} .
$$

Suppose that $\pi-\hat{\alpha}\left(s^{+}\right)-\hat{\beta}\left(s^{+}\right)>\varepsilon /(5 \pi)$, or equivalently that

$$
\pi-\alpha\left(s^{+}\right)-\beta\left(s^{+}\right)<-\frac{\varepsilon}{5 \pi} \text {. }
$$

Under this assumption, we shall show that

$$
\int_{s^{+}}^{3 s_{1}} l^{\prime}(s) d s>L
$$

which is impossible, since $0 \leq l \leq L$. Suppose that $s \geq s^{+}$is good. Since $s^{+}$is also good, (2.2) with $s^{\prime}=s^{+}$and $s^{\prime \prime}=s$ gives that

$$
\begin{aligned}
0 & \leq 2 \pi\left\{2 \pi-\hat{\alpha}(s)-\hat{\beta}(s)-\alpha\left(s^{+}\right)-\beta\left(s^{+}\right)\right\}+2 \int_{T^{1}\left(s^{+}\right) \cup T^{1}(s)}|U(v)| d \lambda(v) \\
& <2 \pi\left\{\pi-\hat{\alpha}(s)-\hat{\beta}(s)-\frac{\varepsilon}{5 \pi}\right\}+\frac{\varepsilon}{5},
\end{aligned}
$$

so that

$$
\hat{\alpha}(s)+\hat{\beta}(s)<\pi-\frac{\varepsilon}{5 \pi}+\frac{\varepsilon}{10 \pi}=\pi-\frac{\varepsilon}{10 \pi} .
$$

Since $\hat{\alpha}(s)$ and $\hat{\beta}(s)$ are both nonnegative, (2.8) implies

$$
|\hat{\alpha}(s)-\hat{\beta}(s)|<\pi-\frac{\varepsilon}{10 \pi} \text {. }
$$

It follows from the first variation of arclength formula that

$$
l^{\prime}(s)=\cos \hat{\alpha}(s)+\cos \hat{\beta}(s)=2 \cos \frac{\hat{\alpha}(s)+\hat{\beta}(s)}{2} \cos \frac{\hat{\alpha}(s)-\hat{\beta}(s)}{2} .
$$

It is clear from (2.8), (2.9) and (2.10) that if $s \geq s^{+}$is good, then

$$
l^{\prime}(s) \geq 2 \sin ^{2} \frac{\varepsilon}{20 \pi} \geq 2\left\{\frac{2}{\pi} \frac{\varepsilon}{20 \pi}\right\}^{2}=\frac{\varepsilon^{2}}{50 \pi^{4}},
$$

since $0<\varepsilon<10 \pi$, and $\sin x$ is convex for $0<x<\pi / 2$. Even if $s$ is not good, we have $l^{\prime}(s) \geq-2$. From these estimates and Lemma 2.5 we 
see that if $0<\varepsilon<10 \pi$, then

$$
\begin{aligned}
\int_{s^{+}}^{3 s_{1}} l^{\prime}(s) d s \geq & \frac{\varepsilon^{2}}{50 \pi^{4}} \text { length }\left\{s \in\left[s^{+}, 3 s_{1}\right]: s \text { is good }\right\} \\
& \quad-2 \text { length }\left\{s \in\left[s^{+}, 3 s_{1}\right]: s \text { is not good }\right\} \\
\geq & \frac{\varepsilon^{2}}{50 \pi^{4}}\left(s_{1}-\sqrt{s_{1}}\right)-2 \sqrt{s_{1}} \\
> & L
\end{aligned}
$$

by (2.6). Since this is impossible, $\pi-\hat{\alpha}\left(s^{+}\right)-\hat{\beta}\left(s^{+}\right) \leq \varepsilon /(5 \pi)$. A similar argument shows that $\pi-\alpha\left(s^{-}\right)-\beta\left(s^{-}\right) \leq \varepsilon /(5 \pi)$. Thus (2.7) is true, which completes the proof of the theorem.

We now show that the above theorem can be viewed as a special case of Conjecture 0.2 .

2.6. Lemma. Suppose $C$ has no conjugate points, curvature bounded from below and bounded cross section. Then every point of $\widetilde{C}$ lies on a geodesic whose Hausdorff distance from $\gamma_{1}$ is bounded.

Proof. It is enough to show that every point of $\Sigma$ lies on a geodesic that does not leave $\Sigma$. Since $C$ has bounded cross section, $\gamma_{1}$ and $\gamma_{2}$ have finite Hausdorff distance. It is obvious from this and Proposition 1.6 that a geodesic that starts at a point in $\Sigma$ stays in $\Sigma$ for all time if and only if it does not cross $\gamma_{1}$. Proposition 1.7 implies that every point of $\Sigma$ lies on a geodesic that does not intersect $\gamma_{1}$.

2.7. Definition. The cylinder $C$ is constricted if there are sequences $p_{n}$ and $q_{n}$ such that $p_{n}$ diverges to one end of $C, q_{n}$ diverges to the other end of $C$ and $\lim _{n \rightarrow \infty} l\left(p_{n}\right)=0=\lim _{n \rightarrow \infty} l\left(q_{n}\right)$.

2.8. Theorem. Let $C$ be a cylinder with curvature bounded from below that is constricted. Then $C$ has conjugate points.

Proof. Assume that $C$ has no conjugate points. We use the same notation as in the proof of Theorem 2.2. Since $C$ is constricted, $\liminf _{s \rightarrow \infty} l(s)$ $=0$. Either there is $s_{0}$ such that $l^{\prime}(s) \leq 0$ for all $s \geq s_{0}$ or there is not. In the first case, $\lim _{s \rightarrow \infty} l(s)=\limsup _{s \rightarrow \infty} l^{\prime}(s)=0$. In the latter case, we can choose $s_{n}^{+} \rightarrow \infty$ such that $l$ has a local minimum at each $s_{n}^{+}$and $l\left(s_{n}^{+}\right) \rightarrow 0$ as $n \rightarrow \infty$. In either case we obtain a sequence $s_{n}^{+} \rightarrow \infty$ such that $l\left(s_{n}^{+}\right) \rightarrow 0$ and $l^{\prime}\left(s_{n}^{+}\right) \rightarrow 0$. In a similar way we can choose a sequence $s_{n}^{-} \rightarrow-\infty$ such that $l\left(s_{n}^{-}\right) \rightarrow 0$ and $l^{\prime}\left(s_{n}^{-}\right) \rightarrow 0$. It is clear from (2.10) that $\hat{\alpha}\left(s_{n}^{+}\right)+\hat{\beta}\left(s_{n}^{+}\right) \rightarrow \pi$ and $\alpha\left(s_{n}^{-}\right)+\beta\left(s_{n}^{-}\right) \rightarrow \pi$ as $n \rightarrow \infty$. By (2.2), we 
have

$$
\begin{aligned}
\int_{T^{1} Q\left(s_{n}^{-}, s_{n}^{+}\right)} U^{2}(v) d \mu(v) \leq & 2 \pi\left\{2 \pi-\hat{\alpha}\left(s_{n}^{+}\right)-\hat{\beta}\left(s_{n}^{+}\right)-\alpha\left(s_{n}^{-}\right)-\beta\left(s_{n}^{-}\right)\right\} \\
& +4 \pi b\left\{l\left(s_{n}^{-}\right)+l\left(s_{n}^{+}\right)\right\} \rightarrow 0
\end{aligned}
$$

as $n \rightarrow \infty$. Hence $\int_{T^{1} C} U^{2}(v) d \mu(v)=0$. It follows as in the proof of Theorem 2.2 that $C$ is flat. But then $l(s)$ is constant, which is impossible if $C$ is constricted.

2.9. Corollary. Let $C$ be a cylinder with curvature bounded from below and finite area. Then $C$ has conjugate points.

\section{Planes with "parallel" geodesics}

This section contains the proof of

3.1. Theorem. Let $P$ be the plane $\mathbb{R}^{2}$ with a Riemannian metric. Suppose that there is a constant $a \geq 1$ such that for every point $p$ and every geodesic $\gamma$ in $P$, there is a geodesic $\beta$ with $\beta(0)=p$ and

$$
\operatorname{dist}\left(\beta\left(t^{\prime}\right), \gamma\right) \leq a \operatorname{dist}(\beta(t), \gamma) \quad \text { for all } t, t^{\prime} .
$$

Then $g$ is flat.

We shall say that the geodesic $\beta$ of $P$ is "weakly parallel" to $\gamma$ if (3.1) holds. Note that if $\beta$ is "weakly parallel" to $\gamma$, then either $\beta$ is a reparametrization of $\gamma$ or $\beta$ does not intersect $\gamma$.

3.2. Lemma. Let $P$ be as in Theorem 3.1. Then the following hold:

(i) $P$ has no conjugate points;

(ii) $y_{-}=y_{+}$along every geodesic;

(iii) $y_{-}(v, t) \leq a y_{-}\left(v, t^{\prime}\right)$ for all $v$ and all $t$ and $t^{\prime}$. In particular $P$ has the bounded asymptote property of [5].

Proof. (i) follows easily from Proposition 1.7.

We now show that (iii) holds whenever $t^{\prime} \leq t$. If not, there are $t_{3}>t_{2}>$ $t_{1}>0$ and an orthogonal Jacobi field along a geodesic $\gamma$ with $\left\|Y\left(t_{2}\right)\right\|>$ $a\left\|Y\left(t_{1}\right)\right\|$ and $Y\left(t_{3}\right)=0$. Thus there is a geodesic $\delta$ with $\delta\left(t_{3}\right)=\gamma\left(t_{3}\right)$ and $\operatorname{dist}\left(\delta\left(t_{2}\right), \gamma\right)>a \operatorname{dist}\left(\delta\left(t_{1}\right), \gamma\right)$. But this is impossible, because one of the geodesics "weakly parallel" to $\gamma$ would have to cross $\delta$ twice.

Next we show that $z(v, t) \rightarrow \infty$ as $t \rightarrow \infty$ for every $v \in T^{1} P$. Since $y_{+}(v, t)=y_{-}(-v,-t)$, it follows from the above that

$$
y_{+}(v, t) \geq a^{-1} y_{+}\left(v, t^{\prime}\right) \quad \text { whenever } t \geq t^{\prime} .
$$


In particular, $y_{+}(v, t) \geq a^{-1}$ whenever $t \geq 0$. It is clear from this and (1.2) that $z(v, t) \rightarrow \infty$ as $t \rightarrow \infty$ if $\int_{0}^{\infty}\left(y_{+}(v, t)\right)^{-2} d t$ diverges. If this integral converges, there is a sequence $t_{n} \rightarrow \infty$ such that $y_{+}\left(v, t_{n}\right) \rightarrow \infty$. Then it is clear from (3.2) that $y_{+}(v, t) \rightarrow \infty$ as $t \rightarrow \infty$, and it follows easily from (1.2) that $z(v, t) \rightarrow \infty$.

Now suppose that (ii) is false. Then we can find $v$ and a solution of the scalar Jacobi equation (1.1) such that $y(0)=1$ and $y_{-}^{\prime}(v, 0)<$ $y^{\prime}(0)<y_{+}^{\prime}(v, 0)$. It is clear that $y(t)>0$ for all $t$ and $y(t) \rightarrow \infty$ as $t \rightarrow \pm \infty$. Thus we can choose $t_{-}<0<t_{+}$and a geodesic $\delta$ such that $\delta\left(t_{-}\right), \delta(0)$ and $\delta\left(t_{+}\right)$are all on the same side of $\gamma_{v}$ and $\operatorname{dist}\left(\delta\left(t_{ \pm}\right), \gamma_{v}\right)>$ $a \operatorname{dist}\left(\delta(0), \gamma_{v}\right)$. But this means that one of the geodesics "weakly parallel" to $\gamma_{v}$ crosses $\delta$ twice, which is impossible. Thus (ii) holds.

It follows easily from (ii) and the fact that (iii) holds when $t^{\prime} \leq t$ that (iii) holds for all $t$ and $t^{\prime}$.

\subsection{Lemma.}

(i) $a^{-1} \leq y_{-}(v, t) \leq a$ for all $v$ and $t$;

(ii) $a^{-3} t \leq z(v, t) \leq a^{3} t$ for all $v$ and $t$;

(iii) there is $B>1$ such that, if $y$ is a Jacobi field along a geodesic $\gamma$ of $P$ and $y(t)>0$ for $t_{1} \leq t \leq t_{2}$, then

$$
B^{-1} \min \left\{y\left(t_{1}\right), y\left(t_{2}\right)\right\} \leq y(t) \leq B \max \left\{y\left(t_{1}\right), y\left(t_{2}\right)\right\},
$$

for $t_{1} \leq t \leq t_{2}$

(iv) if $\gamma$ and $\delta$ are unit speed geodesics with the same initial point and $\varangle(\dot{\gamma}(0), \dot{\delta}(0))=\psi$, then

for all $t \geq 0$.

$$
\frac{\psi t}{2 \pi a^{3}} \leq \operatorname{dist}(\gamma(t), \delta(t)) \leq a^{3} \psi t
$$

Proof. (i) This is immediate from Lemma 3.2.

(ii) This follows from (i) and (1.2).

(iii) Let $z_{i}$ be the scalar Jacobi field along $\gamma$ with $z_{i}\left(t_{i}\right)=0$ and $z_{i}^{\prime}\left(t_{i}\right)=(-1)^{i-1}$. The sign of $z_{i}^{\prime}\left(t_{i}\right)$ is chosen so that $z_{i}(t)>0$ for $t_{1}<t<t_{2}$. Let $j$ denote the element of $\{1,2\}$ that is not $i$. Then

$$
y(t)=\frac{y\left(t_{i}\right)}{y_{-}\left(t_{i}\right)} y_{-}(t)+\frac{y\left(t_{j}\right)-\left\{y\left(t_{i}\right) / y_{-}\left(t_{i}\right)\right\} y_{-}\left(t_{j}\right)}{z_{i}\left(t_{j}\right)} z_{i}(t) \quad \text { for } i=1,2 \text {, }
$$

where $y_{-}$is the stable scalar Jacobi field along $\gamma$. We can choose $i$ so that the coefficient of $z_{i}(t)$ is nonnegative. Then we have, for $t_{1}<t<t_{2}$,

$$
y(t) \geq \frac{y\left(t_{i}\right)}{y_{-}\left(t_{i}\right)} y_{-}(t) \geq a^{-2} \min \left\{y\left(t_{1}\right), y\left(t_{2}\right)\right\},
$$


by (i). Also (i) and (ii) imply that for $t_{1}<t<t_{2}$,

$$
\begin{aligned}
y(t) & \leq \frac{y\left(t_{i}\right)}{y_{-}\left(t_{i}\right)} y_{-}(t)+\frac{y\left(t_{j}\right)}{z_{i}\left(t_{j}\right)} z_{i}(t) \\
& \leq a^{2} y\left(t_{i}\right)+\frac{y\left(t_{j}\right)}{a^{-3}\left(t_{2}-t_{1}\right)} a^{3}\left|t-t_{i}\right| \\
& \leq 2 a^{6} \max \left\{y\left(t_{1}\right), y\left(t_{2}\right)\right\} .
\end{aligned}
$$

Thus we can choose $B=2 a^{6}$.

(iv) The upper bound follows from (ii). For the lower bound, let $r$ and $\theta$ be polar coordinates about $\gamma(0)$, so that $\partial / \partial r$ is the unit vector field pointing away from $\gamma(0)$ and, along each ray starting from $\gamma(0)$, $\partial / \partial \theta$ is the perpendicular Jacobi field with $\|\partial / \partial \theta\|=z$. By (ii), we have $\|\partial / \partial \theta\| \geq a^{-3} t / 2$ outside the circle about $\gamma(0)$ with radius $t / 2$. Thus any curve from $\gamma(t)$ to $\delta(t)$ that lies outside this circle has length at least $a^{-3} \psi t / 2$. But any curve from $\gamma(t)$ to $\delta(t)$ that goes inside this circle has length at least $t$. Since $\psi / \pi \leq 1$, we see that $\operatorname{dist}(\gamma(t), \delta(t)) \geq$ $\psi t /\left(2 a^{3} \pi\right)$.

3.4. Lemma. Suppose that $\beta$ is "weakly parallel" to $\gamma$. Then there is a unique $\varepsilon \in\{-1,1\}$ such that

$$
\operatorname{dist}\left(\beta\left(t^{\prime}\right), \gamma\left(\varepsilon t^{\prime}\right)\right) \leq 3 a \operatorname{dist}(\beta(t), \gamma(\varepsilon t)) \quad \text { for all } t, t^{\prime} .
$$

The proof is based on

3.5. Lemma. Suppose that $\tilde{\beta}$ is "weakly parallel" to $\tilde{\gamma}$. Then there is a unique $\tilde{\varepsilon} \in\{-1,1\}$ such that $\operatorname{dist}(\tilde{\beta}(s), \tilde{\gamma}(\tilde{\varepsilon} s))$ is uniformly bounded. Furthermore

$$
\operatorname{dist}(\tilde{\beta}(s), \tilde{\gamma}(\tilde{\varepsilon} s)) \leq 3 a \operatorname{dist}(\tilde{\beta}(0), \tilde{\gamma}(0)) \quad \text { for all } s .
$$

Proof. Lemma 3.2(i) tells us that every geodesic in $P$ is minimizing. It follows that $\operatorname{dist}(\tilde{\beta}(s), \tilde{\gamma}(s))$ and $\operatorname{dist}(\tilde{\beta}(s), \tilde{\gamma}(-s))$ cannot both be uniformly bounded, for then we would have $\operatorname{dist}(\tilde{\gamma}(s), \tilde{\gamma}(-s))$ uniformly bounded, which is impossible for a minimizing geodesic.

Since $\tilde{\beta}$ is "weakly parallel" to $\tilde{\gamma}$, there is a function $\tau: \mathbb{R} \rightarrow \mathbb{R}$ with $\tau(0)=0$ such that for all $s$,

$$
\operatorname{dist}(\tilde{\beta}(s), \tilde{\gamma}(\tau(s))) \leq a \operatorname{dist}(\tilde{\beta}(0), \tilde{\gamma}) \leq a \operatorname{dist}(\tilde{\beta}(0), \tilde{\gamma}(0)) .
$$


For any $s_{1}$ and $s_{2}$, we have

$$
\begin{aligned}
\left|\tau\left(s_{1}\right)-\tau\left(s_{2}\right)\right| & =\operatorname{dist}\left(\tilde{\gamma}\left(\tau\left(s_{1}\right)\right), \tilde{\gamma}\left(\tau\left(s_{2}\right)\right)\right) \\
\leq & \operatorname{dist}\left(\tilde{\gamma}\left(\tau\left(s_{1}\right)\right), \tilde{\beta}\left(s_{1}\right)\right)+\operatorname{dist}\left(\tilde{\beta}\left(s_{1}\right), \tilde{\beta}\left(s_{2}\right)\right) \\
& \quad+\operatorname{dist}\left(\tilde{\beta}\left(s_{2}\right), \tilde{\gamma}\left(\tau\left(s_{2}\right)\right)\right) \\
\leq & \left|s_{1}-s_{2}\right|+2 a \operatorname{dist}(\tilde{\beta}(0), \tilde{\gamma}(0)),
\end{aligned}
$$

and similarly

$$
\left|s_{1}-s_{2}\right| \leq\left|\tau\left(s_{1}\right)-\tau\left(s_{2}\right)\right|+2 a \operatorname{dist}(\tilde{\beta}(0), \tilde{\gamma}(0)) .
$$

It follows easily that there are $\tilde{\varepsilon} \in\{-1,1\}$ and $c \in \mathbb{R}$ such that

$$
|\tilde{\varepsilon} s+c-\tau(s)| \leq a \operatorname{dist}(\tilde{\beta}(0), \tilde{\gamma}(0)) \quad \text { for all } s .
$$

Setting $s=0$ gives

$$
|c| \leq a \operatorname{dist}(\tilde{\beta}(0), \tilde{\gamma}(0)) .
$$

Combining (3.4), (3.5) and (3.6) shows that for all $s$,

$$
\begin{aligned}
\operatorname{dist}(\tilde{\beta}(s), \tilde{\gamma}(\tilde{\varepsilon} s)) \leq & \operatorname{dist}(\tilde{\beta}(s), \tilde{\gamma}(\tau(s))) \\
& +\operatorname{dist}(\tilde{\gamma}(\tau(s)), \tilde{\gamma}(\tilde{\varepsilon} s+c))+\operatorname{dist}(\tilde{\gamma}(\tilde{\varepsilon} s+c), \tilde{\gamma}(\tilde{\varepsilon} s)) \\
\leq & 3 a \operatorname{dist}(\tilde{\beta}(0), \tilde{\gamma}(0)) .
\end{aligned}
$$

Proof of Lemma 3.4. By Lemma 3.5, there is a unique $\varepsilon \in\{-1,1\}$ such that

$$
\operatorname{dist}(\beta(t), \gamma(\varepsilon t)) \leq 3 a \operatorname{dist}(\beta(0), \gamma(0)) \quad \text { for all } t .
$$

Now consider the geodesics $\tilde{\beta}_{t}$ and $\tilde{\gamma}_{t}$ defined by $\tilde{\beta}_{t}(s)=\beta(t+s)$ and $\tilde{\gamma}_{t}(s)=\gamma(\varepsilon t+\varepsilon s)$. The geodesic $\tilde{\beta}_{t}$ is "weakly parallel" to $\tilde{\gamma}_{t}$ and it is clear from (3.7) that, for each $t, \operatorname{dist}\left(\tilde{\beta}_{t}(s), \tilde{\gamma}_{t}(s)\right)$ is uniformly bounded for all $s$. It follows from Lemma 3.5 that for all $s$ and $t$,

$$
\operatorname{dist}\left(\tilde{\beta}_{t}(s), \tilde{\gamma}_{t}(s)\right) \leq 3 a \operatorname{dist}\left(\tilde{\beta}_{t}(0), \tilde{\gamma}_{t}(0)\right)=\operatorname{dist}(\beta(t), \gamma(\varepsilon t)) .
$$

Setting $s=t^{\prime}-t$ gives us (3.3).

3.6. Definition. The geodesics $\alpha$ and $\beta$ are "parallel" if

$$
\operatorname{dist}\left(\alpha\left(t^{\prime}\right), \beta\left(t^{\prime}\right)\right) \leq 3 a \operatorname{dist}(\alpha(t), \beta(t)) \quad \text { for all } t, t^{\prime}
$$

and "antiparallel" if $\alpha$ is "parallel" to $t \mapsto \beta(-t)$.

It follows from Lemma 3.4 that for every point $p$ and every geodesic $\gamma$ of $P$ there is a geodesic $\beta$ with $\beta(0)=p$ that is "parallel" to $\gamma$. Lemma 3.3(iv) shows that this uniquely determines $\beta$. Moreover $\beta$ is (up to reparametrization) the unique geodesic through $p$ that does not cross $\gamma$ 
transversally. We see that "weak parallelism" is an equivalence relation (in particular symmetric) and two geodesics are "weakly parallel" if and only if they are "parallel" or "antiparallel". We also see that "parallelism" is a continuous relation in the following sense: if $v_{n} \rightarrow v$ in $T^{1} P$ and each $\gamma_{v_{n}}$ is "parallel" to a given geodesic $\gamma$, then $\gamma_{v}$ is "parallel" to $\gamma$.

We use this to introduce coordinates on $P$. Choose a point $p_{0}$ to be the origin. Choose geodesics $\gamma_{1}$ and $\gamma_{2}$ with $\gamma_{1}(0)=p_{0}=\gamma_{2}(0)$. Let $\Gamma_{2}(r, \cdot)$ be the geodesic "parallel" to $\gamma_{2}$ with $\Gamma_{2}(r, 0)=\gamma_{1}(r)$. Let $\Gamma_{1}(s, \cdot)$ be the geodesic "parallel" to $\gamma_{1}$ with $\Gamma_{1}(s, 0)=\gamma_{2}(s)$ and $\dot{\Gamma}_{1}(s, 0)$ on the same side of $\gamma_{2}$ as $\dot{\gamma}_{1}(0)$. Define the coordinates $x^{1}$ and $x^{2}$ so that $\Gamma_{2}(r, \cdot)$ and $\Gamma_{1}(s, \cdot)$ meet at the point with coordinates $(r, s)$.

These coordinates are $C^{1}$. To see this, note that geodesics asymptotic (in the sense defined in $\S 1$ ) to $\gamma_{2}$ do not intersect $\gamma_{2}$ transversally. It follows easily that $\Gamma_{2}(r, \cdot)$ is the geodesic through $\gamma_{1}(r)$ that is asymptotic to $\gamma_{2}$. Since $P$ has bounded asymptote by Lemma 3.2(iii), Proposition 5 and Theorem 1 of [5] imply that $\dot{\Gamma}_{2}(r, 0)$ is a $C^{1}$ function of $r$; similarly $\dot{\Gamma}_{1}(s, 0)$ is a $C^{1}$ function of $s$. It follows easily that the coordinates are $C^{1}$

3.7. Lemma. There is $\eta$ such that $0<\eta \leq \pi / 2$ and

$$
\eta \leq \varangle\left(\partial / \partial x^{1}, \partial / \partial x^{2}\right) \leq \pi-\eta
$$

everywhere in $P$.

Proof. Let $\psi(p)$ be the angle between $\partial / \partial x^{1}(p)$ and $\partial / \partial x^{2}(p)$. For $i=1,2$ and $t>0$, let $q_{i}(p, t)$ be the point obtained by moving distance $t$ in the $x^{i}$-direction from $p$. Note that $\psi\left(p_{0}\right)=\pi / 2$ and $q_{i}\left(p_{0}, t\right)=$ $\gamma_{i}(t)$. Let

$$
d_{0}(t)=\operatorname{dist}\left(\gamma_{1}(t), \gamma_{2}(t)\right) \quad \text { and } \quad d_{p}(t)=\operatorname{dist}\left(q_{1}(p, t), q_{2}(p, t)\right) .
$$

It follows from Lemma 3.3(iv) that

$$
d_{0}(t) \geq \frac{t}{4 a^{3}} \quad \text { and } \quad d_{p}(t) \leq a^{3} \psi(p) t \quad \text { for all } t>0 .
$$

On the other hand, $\left|d_{0}(t)-d_{p}(t)\right|$ is uniformly bounded for all $t$, since

$$
\left|d_{0}(t)-d_{p}(t)\right| \leq \operatorname{dist}\left(\gamma_{1}(t), q_{1}(p, t)\right)+\operatorname{dist}\left(\gamma_{2}(t), q_{2}(p, t)\right)
$$

and $\gamma_{i}(\cdot)$ and $q_{i}(p, \cdot)$ are "parallel" geodesics. Thus $a^{3} \psi(p) \geq 1 /\left(4 a^{3}\right)$, and therefore $\psi(p) \geq 1 /\left(4 a^{6}\right)$. A similar argument can be applied to the angle between $\partial / \partial x^{1}$ and $-\partial / \partial x^{2}$ to show that $\pi-\psi(p) \geq 1 /\left(4 a^{6}\right)$. 
3.8. Lemma. Let $A=a / \sin \eta$, and $y_{i}$ be the length of the projection of $\partial / \partial x^{i}$ onto the direction orthogonal to the curves $x^{i}=$ const. Then the following hold for all $p \in P$ and $i=1,2$.

(i) $A^{-1} \leq y_{i}(p) \leq a$;

(ii) $a^{-1} \leq\left\|\partial / \partial x^{i}(p)\right\| \leq A$;

(iii) $a^{-1} \leq\left\|\operatorname{grad}_{p} x^{i}\right\| \leq A$.

Proof. Let $j$ be the element of $\{1,2\}$ that is not $i$. Observe that if $\beta_{j}$ is a geodesic "parallel" to $\gamma_{j}$, then $y_{i} \circ \beta_{j}$ is a scalar Jacobi field along $\beta_{j}$ that never vanishes. Since $y_{+}=y_{-}$along $\beta_{j}, y_{i} \circ \beta_{j}$ is a multiple of the stable solution $y_{-}$. It follows from Lemma 3.2(ii) that if $t_{0}$ is the time when $\beta_{j}$ crosses $\gamma_{i}$, then

$$
a^{-1} y_{i} \circ \beta_{j}\left(t_{0}\right) \leq y_{i} \circ \beta_{j}(t) \leq a y_{i} \circ \beta_{j}\left(t_{0}\right) \quad \text { for all } t .
$$

Thus by Lemma 3.7 we have

$$
\sin \eta\left\|\partial / \partial x^{i}(p)\right\| \leq y_{i}(p) \leq\left\|\partial / \partial x^{i}(p)\right\| \quad \text { for all } p \in P .
$$

Since we chose $x^{i}$ so that $\left\|\partial / \partial x^{i}\right\| \equiv 1$ along $\gamma_{i}$, part (i) of the lemma follows from (3.8) and (3.9). Part (ii) follows from (i) and (3.9). Part (iii) follows from (i), since $\left\|\operatorname{grad}_{p} x^{i}\right\|=y_{i}(p)^{-1}$.

3.9. Definition. Let $\mathscr{S}(r)=\left\{p:-r \leq x^{1}(p), x^{2}(p) \leq r\right\}$ be the "square" defined by the coordinates.

3.10. Lemma. For any $r \geq 0$, the "square" $\mathscr{S}(r)$ satisfies

$$
\lambda\left(\partial^{1} \mathscr{S}(r)\right) \leq 16 \pi A r .
$$

If $0 \leq r^{\prime} \leq r^{\prime \prime}$ and $\phi: T^{1} \mathscr{S}\left(r^{\prime \prime}\right) \rightarrow[0, \infty)$ is integrable, then

$$
\begin{aligned}
\frac{1}{A} \int_{r^{\prime}}^{r^{\prime \prime}} \int_{\partial^{1} \mathscr{S}(r)} \phi(v) d \lambda(v) d r & \leq \int_{T^{1}\left(\mathscr{S}\left(r^{\prime \prime}\right) \backslash \mathscr{S}\left(r^{\prime}\right)\right)} \phi(v) d \mu(v) \\
& \leq A \int_{r^{\prime}}^{r^{\prime \prime}} \int_{\partial^{1} \mathscr{S}(r)} \phi(v) d \lambda(v) d r .
\end{aligned}
$$

Proof. This follows easily from the previous lemma.

3.11. Proposition. $U \in L^{2}(P)$.

Proof. Let $f(r)=\int_{\partial^{1} \mathscr{S}(r)} U^{2}(v) d \lambda(v)$. Then by Lemma 3.10 and Lemma 1.3 applied to $\mathscr{S}(R)$ we see that 


$$
\begin{aligned}
A^{-1} \int_{0}^{R} f(r) d r & \leq \int_{T^{1} \mathscr{S}(R)} U^{2}(v) d \mu(v) \\
& \leq-2 \pi \int_{\mathscr{S}(R)} K(p) d \nu(p)+2 \int_{\partial^{1} \mathscr{S}(R)}|U(v)| d \lambda(v) .
\end{aligned}
$$

Since $\mathscr{S}(R)$ has geodesic sides and four interior angles between 0 and $\pi$,

$$
-\int_{\mathscr{S}(R)} K(p) d \nu(p) \leq 2 \pi
$$

by the Gauss-Bonnet theorem. On the other hand, Hölder's inequality and Lemma 3.10 imply that

$$
\begin{aligned}
\int_{\partial^{1} \mathscr{S}(R)}|U(v)| d \lambda(v) & \leq\left\{\int_{\partial^{1} \mathscr{S}(R)} 1 d \lambda(v)\right\}^{1 / 2}\left\{\int_{\partial^{1} \mathscr{S}(R)} U^{2}(v) d \lambda(v)\right\}^{1 / 2} \\
& \leq \sqrt{16 \pi A R} \sqrt{f(R)}
\end{aligned}
$$

Hence

$$
\int_{0}^{R} f(r) d r \leq 4 \pi^{2} A+8 A \sqrt{\pi A} \sqrt{R f(R)} .
$$

Lemma 3.12 below now shows that $f \in L^{1}([0, \infty))$. It follows from Lemma 3.10 that

$$
\int_{T^{1} P} U^{2}(v) d \mu(v) \leq A \int_{0}^{\infty} f(r) d r<\infty
$$

3.12. Lemma. Let $f:[0, \infty) \rightarrow[0, \infty)$. Suppose there are $C_{1}, C_{2}>$ 0 such that

$$
\int_{0}^{R} f(r) d r \leq C_{1}+C_{2} \sqrt{R f(R)} \quad \text { for all } R \geq 0 .
$$

Then

$$
\int_{0}^{R} f(r) d r \leq C_{1} \quad \text { for all } R \geq 0
$$

Proof. Let $F(R)=\int_{0}^{R} f(r) d r-C_{1}$. Suppose that $F\left(R_{0}\right)>0$. Then $F^{2}(R) \leq C_{2}^{2} R F^{\prime}(R)$ and $\quad F(R)>0 \quad$ for all $R \geq R_{0}$ 
and hence

$$
\ln R \leq \ln R_{0}+\frac{C_{2}^{2}}{F\left(R_{0}\right)}-\frac{C_{2}^{2}}{F(R)} \leq \ln R_{0}+\frac{C_{2}^{2}}{F\left(R_{0}\right)} \quad \text { for all } R \geq R_{0},
$$

which is impossible.

3.13. Lemma. $\left|x^{2}\left(\Gamma_{2}(r, s)\right)\right| \geq A^{-1}|s|$ for all $r$ and $s$.

Proof. This follows from Lemma 3.8(ii), since $\Gamma_{2}(r, \cdot)$ is parametrized by arclength and is always tangent to $\partial / \partial x^{2}$.

3.14. Lemma. Suppose $r^{\prime}<r^{\prime \prime}$. Then for all $s$,

$$
\operatorname{dist}\left(\Gamma_{2}\left(r^{\prime}, s\right), \Gamma_{2}\left(r^{\prime \prime}, s\right)\right) \leq 3 a\left(r^{\prime \prime}-r^{\prime}\right) .
$$

Proof. $\Gamma_{2}\left(r^{\prime}, \cdot\right)$ is "parallel" to $\Gamma_{2}\left(r^{\prime \prime}, \cdot\right)$ and $\operatorname{dist}\left(\Gamma_{2}\left(r^{\prime}, 0\right), \Gamma_{2}\left(r^{\prime \prime}, 0\right)\right)$ $=r^{\prime \prime}-r^{\prime}$.

3.15. Lemma. There is $\Theta>0$ such that for any $r^{\prime}, r^{\prime \prime}$ and $s$ with $r^{\prime}<r^{\prime \prime}$, the geodesic through $\Gamma_{2}\left(r^{\prime}, s\right)$ and $\Gamma_{2}\left(r^{\prime \prime}, s\right)$ crosses $\Gamma_{2}\left(r^{\prime}, \cdot\right)$ and $\Gamma_{2}\left(r^{\prime \prime}, \cdot\right)$ with angle at least $\Theta$.

Proof. Let $\sigma$ be the geodesic segment from $\Gamma_{2}\left(r^{\prime}, s\right)$ to $\Gamma_{2}\left(r^{\prime \prime}, s\right)$. Then length $(\sigma) \leq 3 a\left(r^{\prime \prime}-r^{\prime}\right)$ by the previous lemma. Suppose that $\sigma$ makes angle less than $1 /\left(6 a^{4} A\right)$ with $\Gamma_{2}\left(r^{\prime}, \cdot\right)$ or $\Gamma_{2}\left(r^{\prime \prime}, \cdot\right)$. Then by Lemma 3.3(iv), there are points $p^{\prime}$ on $\Gamma_{2}\left(r^{\prime}, \cdot\right)$ and $p^{\prime \prime}$ on $\Gamma_{2}\left(r^{\prime \prime}, \cdot\right)$ with $\operatorname{dist}\left(p^{\prime}, p^{\prime \prime}\right) \leq(1 / 2 A)\left(r^{\prime \prime}-r^{\prime}\right)$. From this and Lemma 3.8(iii) it follows that

$$
\left|x^{2}\left(p^{\prime \prime}\right)-x^{2}\left(p^{\prime}\right)\right| \leq A \frac{1}{2 A}\left(r^{\prime \prime}-r^{\prime}\right)=\frac{1}{2}\left(r^{\prime \prime}-r^{\prime}\right),
$$

which is impossible, since $x^{2}\left(p^{\prime \prime}\right)=r^{\prime \prime}$ and $x^{2}\left(p^{\prime}\right)=r^{\prime}$.

3.16. Proposition. Given $r_{0}>0$ and $\varepsilon \in(0,10 \pi)$, there is a set $\mathscr{Q}$ such that $\mathscr{Q} \supseteq \mathscr{S}\left(r_{0}\right)$ and $\int_{T^{1} \mathscr{Q}} U^{2}(v) d \mu(v) \leq \varepsilon$.

Proof. The set $\mathscr{Q}$ will be the convex hull of four points, $\Gamma_{2}\left(r^{+}, s^{+}\right)$, $\Gamma_{2}\left(r^{-}, s^{+}\right), \Gamma_{2}\left(r^{-}, s^{-}\right)$and $\Gamma_{2}\left(r^{+}, s^{-}\right)$, with $r^{-}<-r_{0}, r^{+}>r_{0}, s^{+}>$ $A r_{0}$ and $s^{-}<-A r_{0}$. It is obvious from the first two inequalities that $\mathscr{Q}$ will contain $\mathscr{S}\left(r_{0}\right)$ if neither the side joining $\Gamma_{2}\left(r^{+}, s^{+}\right)$to $\Gamma_{2}\left(r^{+}, s^{-}\right)$ nor the side joining $\Gamma_{2}\left(r^{-}, s^{+}\right)$to $\Gamma_{2}\left(r^{-}, s^{-}\right)$intersects $\mathscr{S}\left(r_{0}\right)$. But this is clear, because the coordinate $x^{2}$ is a monotone function along these edges (in fact along any geodesic), and it follows from Lemma 3.13 that $\left|x^{2}\right|>r_{0}$ at all four corners of $\mathscr{Q}$.

Choose $\rho>1$ large enough so that

$$
\frac{\varepsilon^{2}}{50 \pi^{4}}(\rho-3)-2>12
$$


Choose $r_{1} \geq r_{0}$ large enough so that

$$
\int_{T^{1} \mathscr{S}\left(r_{1} / 2\right)} U^{2}(v) d \mu(v)>\int_{T^{1} P} U^{2}(v) d \mu(v)-\frac{\varepsilon^{2}}{6400 A^{4} \pi \rho}
$$

and

$$
\int_{T^{1} \mathscr{S}\left(r_{1} / 2\right)} U^{2}(v) d \mu(v)>\int_{T^{1} P} U^{2}(v) d \mu(v)-\frac{\varepsilon^{2} \sin ^{2} \Theta}{1600 \cdot 24 B^{3} a \pi \rho},
$$

where the constants $A, B$ and $\Theta$ are defined in Lemma 3.8, Lemma 3.3(iii) and Lemma 3.15 respectively.

We now choose $r^{+}$. For $r^{\prime} \leq r^{\prime \prime}$, let

$$
\mathscr{R}\left(r^{\prime}, r^{\prime \prime}\right)=\left\{p: r^{\prime} \leq x^{1}(p) \leq r^{\prime \prime} \text { and }-\rho r_{1} \leq x^{2}(p) \leq \rho r_{1}\right\}
$$

and let $\mathscr{V}(r)$ be the set of all unit vectors with footpoint on the geodesic segment $\mathscr{R}(r, r)$ which joins $\Gamma_{2}\left(r,-\rho r_{1}\right)$ to $\Gamma_{2}\left(r, \rho r_{1}\right)$. We see from Lemma 3.8 that

$$
\lambda(\mathscr{V}(r)) \leq 4 \pi \rho A r \quad \text { for all } r
$$

and, if $r^{\prime} \leq r^{\prime \prime}$ and $\phi: T^{1} \mathscr{R}\left(r^{\prime}, r^{\prime \prime}\right) \rightarrow[0, \infty)$ is integrable, then

$$
\frac{1}{A} \int_{r^{\prime}}^{r^{\prime \prime}} \int_{\mathscr{V}(r)} \phi(v) d \lambda(v) d r \leq \int_{T^{1} \mathscr{R}\left(r^{\prime}, r^{\prime \prime}\right)} \phi(v) d \mu(v) \leq A \int_{r^{\prime}}^{r^{\prime \prime}} \int_{\mathscr{V}(r)} \phi(v) d \lambda(v) d r
$$
Since

Choose $r^{+} \in\left[r_{1}, 2 r_{1}\right]$ so that $\int_{\mathscr{V}\left(r^{+}\right)}|U(v)| d \lambda(v)$ is as small as possible.

$$
\mathscr{R}\left(r_{1}, 2 r_{1}\right) \cap \mathscr{S}\left(r_{1} / 2\right)=\varnothing
$$


we obtain

$$
\begin{aligned}
\int_{\mathscr{V}\left(r^{+}\right)} & |U(v)| d \lambda(v) \leq \frac{1}{r_{1}} \int_{r_{1}}^{2 r_{1}} \int_{\mathscr{V}(r)}|U(v)| d \lambda(v) d r \\
& \leq \frac{A}{r_{1}} \int_{\mathscr{R}\left(r_{1}, 2 r_{1}\right)}|U(v)| d \mu(v) \\
& \leq \frac{A}{r_{1}}\left\{\int_{\mathscr{R}\left(r_{1}, 2 r_{1}\right)} U^{2}(v) d \mu(v)\right\}^{1 / 2}\left\{\int_{\mathscr{R}\left(r_{1}, 2 r_{1}\right)} 1 d \mu(v)\right\}^{1 / 2} \\
& \leq \frac{A}{r_{1}}\left\{\int_{\mathscr{R}\left(r_{1}, 2 r_{1}\right)} U^{2}(v) d \mu(v)\right\}^{1 / 2}\left\{\int_{r_{1}}^{2 r_{1}} \int_{\mathscr{V}(r)} 1 d \lambda(v) d r\right\}^{1 / 2} \\
& \leq \frac{A}{r_{1}} \frac{\varepsilon}{80 A^{2} \sqrt{\pi \rho}} \sqrt{A \cdot r_{1} \cdot 4 \pi \rho A r_{1}} \text { by }(3.11) \\
& =\frac{\varepsilon}{40} .
\end{aligned}
$$

In a similar way, we choose $r^{-} \in\left[-2 r_{1},-r_{1}\right]$ so that

$$
\int_{\mathscr{V}\left(r^{-}\right)}|U(v)| d \lambda(v) \leq \frac{\varepsilon}{40} .
$$

Now we prepare to choose $s^{+}$and $s^{-}$. Let $\gamma_{-}=\Gamma_{2}\left(r^{-}, \cdot\right)$ and $\gamma_{+}=$ $\Gamma_{2}\left(r^{+}, \cdot\right)$. Let $l(s)=\operatorname{dist}\left(\gamma_{-}(s), \gamma_{+}(s)\right)$ and let $c_{s}$ be the geodesic segment from $\gamma_{-}(s)$ to $\gamma_{+}(s)$, parametrized by arclength so that $c_{s}(0)=\gamma_{-}(s)$ and $c_{s}(l(s))=\gamma_{+}(s)$. Let $\mathscr{T}(s)$ be the set of all unit vectors with footpoint on $c_{s}$. Observe that, by Lemma 3.14 we have

$$
l(s) \leq 3 a \cdot\left(r^{+}-r^{-}\right) \leq 12 a r_{1} \quad \text { and } \quad \lambda\left(\mathscr{T}^{1}(s)\right) \leq 24 \pi a r_{1}
$$

We shall say that $s$ is good if

$$
\int_{\mathscr{T}^{-1}(s)}|U(v)| d \lambda(v) \leq \frac{\varepsilon}{40} .
$$


3.17. Definition. If $s^{\prime} \leq s^{\prime \prime}$, let $\mathscr{Q}\left(s^{\prime}, s^{\prime \prime}\right)$ be the closed set bounded by $\gamma_{-}, \gamma_{+}, c_{s^{\prime}}$ and $c_{s^{\prime \prime}}$.

3.18. Lemma. If $s^{\prime} \leq s^{\prime \prime}$ and $\phi: T^{1} \mathscr{Q}\left(s^{\prime}, s^{\prime \prime}\right) \rightarrow[0, \infty)$ is integrable, then

$$
\begin{aligned}
B^{-1} \sin \Theta \int_{s^{\prime}}^{s^{\prime \prime}} \int_{\mathscr{T}^{1}(s)} \phi(v) d \lambda(v) d s & \leq \int_{T^{1} \mathscr{Q}\left(s^{\prime}, s^{\prime \prime}\right)} \phi(v) d \mu(v) \\
& \leq B \int_{s^{\prime}}^{s^{\prime \prime}} \int_{\mathscr{T}^{1}(s)} \phi(v) d \lambda(v) d s,
\end{aligned}
$$

where $B$ is the constant defined in Lemma 3.3(iii), and $\Theta$ is the angle defined in Lemma 3.15.

Proof. Set $\psi(s, t)=c_{s}(t)$ and let $y_{s}(t)$ be the length of the projection of $(\partial \psi / \partial s)(s, t)$ onto the direction orthogonal to $c_{s}$. Then $y_{s}$ is a scalar Jacobi field along $c_{s}$ that does not vanish for $0 \leq t \leq l(s)$. It suffices to show that for every $s$, we have $B^{-1} \sin \Theta \leq y_{s}(t) \leq B$ for $0 \leq t \leq l(s)$.

Since $\dot{\gamma}_{-}(s)=(\partial \psi / \partial s)(s, 0)$ and

$$
\dot{\gamma}_{+}(s)=\frac{d}{d s}\left(\psi(s, l(s))=\frac{\partial \psi}{\partial s}(s, l(s))+l^{\prime}(s) \dot{c}_{s}(l(s)),\right.
$$

$y_{s}(0)$ and $y_{s}(l(s))$ are the components orthogonal to $c_{s}$ of the unit vectors $\dot{\gamma}_{-}(s)$ and $\dot{\gamma}_{+}(s)$. It follows from Lemma 3.15 that

$$
\sin \Theta \leq y_{s}(0) \leq 1 \text { and } \sin \Theta \leq y_{s}(l(s)) \leq 1 .
$$

Thus by Lemma 3.3(iii), $B^{-1} \sin \Theta \leq y_{s}(t) \leq B$ for $0 \leq t \leq l(s)$. q.e.d. Choose $s_{1}$ so that

$$
s_{1}>A r_{1}
$$

It is clear from Lemma 3.13 that $\mathscr{Q}\left(-s_{1}, s_{1}\right) \supseteq \mathscr{S}\left(r_{1}\right)$. Hence $\mathscr{Q}\left(s_{1}, \rho s_{1}\right)$, $\mathscr{Q}\left(-\rho s_{1},-s_{1}\right)$ and $\mathscr{S}\left(r_{1} / 2\right)$ are pairwise disjoint.

3.19. Lemma. Both of the sets $\left\{s \in\left[s_{1}, \rho s_{1}\right]: s\right.$ is not good $\}$ and $\left\{s \in\left[-\rho s_{1},-s_{1}\right]: s\right.$ is not good $\}$ have length less than $s_{1}$. 
Proof. We consider the first set. Since $\mathscr{Q}\left(s_{1}, \rho s_{1}\right) \cap \mathscr{S}\left(r_{1} / 2\right)=\varnothing$,

$$
\begin{aligned}
B^{-1} & \sin \Theta \cdot \frac{\varepsilon}{40} \cdot \text { length }\left\{s \in\left[s_{1}, \rho s_{1}\right]: s \text { is not good }\right\} \\
& \leq B^{-1} \sin \Theta \int_{s_{1}}^{\rho s_{1}} \int_{\mathscr{T}^{1}(s)}|U(v)| d \lambda(v) d s \quad \text { by Lemma } 3.18 \\
& \leq \int_{T^{1} \mathscr{Q}\left(s_{1}, \rho s_{1}\right)}|U(v)| d \mu(v) \\
& \leq\left\{\int_{T^{1} \mathscr{Q}\left(s_{1}, \rho s_{1}\right)} U^{2}(v) d \mu(v)\right\}^{1 / 2}\left\{\int_{T^{1} \mathscr{Q}\left(s_{1}, \rho s_{1}\right)} 1 d \mu(v)\right\}^{1 / 2} \\
& <\frac{\varepsilon B^{-1} \sin \Theta}{40 \sqrt{24 \pi \rho a \bar{B}}} \sqrt{B \cdot \rho s_{1} \cdot 24 \pi a s_{1}} \begin{array}{c}
\text { by }(3.12),(3.13) \\
\text { and Lemma 3.18 }
\end{array} \\
& =B^{-1} \sin \Theta \cdot \frac{\varepsilon}{40} \cdot s_{1} .
\end{aligned}
$$

This completes the proof for the first set; the other case is similar.

The previous lemma shows that we can choose $s^{+} \in\left[s_{1}, 2 s_{1}\right]$ and $s^{-} \in$ $\left[-2 s_{1},-s_{1}\right]$ so that they are good; our desired set $\mathscr{Q}$ is $\mathscr{Q}\left(s^{-}, s^{+}\right)$.

Let $\alpha(s)=\varangle\left(\dot{c}_{s}(0), \dot{\gamma}_{-}(s)\right)$ and $\hat{\alpha}(s)=\varangle\left(\dot{c}_{s}(0),-\dot{\gamma}_{-}(s)\right)=\pi-\alpha(s)$. Let $\beta(s)=\varangle\left(-\dot{c}_{s}(l(s)), \dot{\gamma}_{+}(s)\right)$ and $\hat{\beta}(s)=\varangle\left(-\dot{c}_{s}(l(s)),-\dot{\gamma}_{+}(s)\right)=\pi-$ $\alpha(s)$. Since $\mathscr{Q}\left(s^{\prime}, s^{\prime \prime}\right)$ is a geodesic quadrilateral with interior angles $\hat{\alpha}\left(s^{\prime \prime}\right), \hat{\beta}\left(s^{\prime \prime}\right), \alpha\left(s^{\prime}\right)$ and $\beta\left(s^{\prime}\right)$, it follows from the Gauss-Bonnet theorem that

$$
-\int_{\mathscr{Q}\left(s^{\prime}, s^{\prime \prime}\right)} K(p) d \nu(p)=\left\{2 \pi-\hat{\alpha}\left(s^{\prime \prime}\right)-\hat{\beta}\left(s^{\prime \prime}\right)-\alpha\left(s^{\prime}\right)-\beta\left(s^{\prime}\right)\right\} .
$$

Note that $\partial^{1} \mathscr{Q}\left(s^{\prime}, s^{\prime \prime}\right) \subseteq \mathscr{V}\left(r^{\prime \prime}\right) \cup \mathscr{V}\left(r^{\prime}\right) \cup \mathscr{T}^{1}\left(s^{\prime}\right) \cup \mathscr{T}^{1}\left(s^{\prime \prime}\right)$. We see from our choice of $r^{-}$and $r^{+}$, that if $s^{\prime}$ and $s^{\prime \prime}$ are both good, then

$$
\int_{\partial^{\prime} \mathscr{Q}\left(s^{\prime}, s^{\prime \prime}\right)}|U(v)| d \lambda(v) \leq \frac{\varepsilon}{10},
$$

which together with Lemma 1.3 implies that if $s^{\prime}$ and $s^{\prime \prime}$ are both good, then

$$
\int_{T^{\prime} \mathscr{Q}\left(s^{\prime}, s^{\prime \prime}\right)} U^{2}(v) d \mu(v) \leq 2 \pi\left\{2 \pi-\hat{\alpha}\left(s^{\prime \prime}\right)-\hat{\beta}\left(s^{\prime \prime}\right)-\alpha\left(s^{\prime}\right)-\beta\left(s^{\prime}\right)\right\}+\frac{\varepsilon}{5} .
$$


In particular, we will have $\int_{T^{1} \mathscr{Q}} U^{2}(v) d \mu(v) \leq \varepsilon$ if

$$
\pi-\hat{\alpha}\left(s^{+}\right)-\hat{\beta}\left(s^{+}\right) \leq \frac{\varepsilon}{5 \pi} \quad \text { and } \quad \pi-\alpha\left(s^{-}\right)-\beta\left(s^{-}\right) \leq \frac{\varepsilon}{5 \pi} .
$$

Suppose that $\pi-\hat{\alpha}\left(s^{+}\right)-\hat{\beta}\left(s^{+}\right)>\varepsilon /(5 \pi)$, or equivalently that

$$
\pi-\alpha\left(s^{+}\right)-\beta\left(s^{+}\right)<-\frac{\varepsilon}{5 \pi} \text {. }
$$

Under this assumption, we shall show that

$$
\int_{s^{+}}^{\rho s_{1}} l^{\prime}(s) d s>12 a r_{1}
$$

which contradicts (3.13). Suppose that $s \in\left[s^{+}, \rho s_{1}\right]$ is good. Since $s^{+}$is also good, (3.15) with $s^{\prime}=s^{+}$and $s^{\prime \prime}=s$ shows that

$$
\begin{aligned}
0 & \leq 2 \pi\left\{2 \pi-\hat{\alpha}(s)-\hat{\beta}(s)-\alpha\left(s^{+}\right)-\beta\left(s^{+}\right)\right\}+\frac{\varepsilon}{5} \\
& <2 \pi\left\{\pi-\hat{\alpha}(s)-\hat{\beta}(s)-\frac{\varepsilon}{5 \pi}\right\}+\frac{\varepsilon}{5}
\end{aligned}
$$

and hence

$$
\hat{\alpha}(s)+\hat{\beta}(s)>\pi+\frac{\varepsilon}{5 \pi}-\frac{\varepsilon}{10 \pi}=\pi+\frac{\varepsilon}{10 \pi} .
$$

As in $\S 2$, we have $l^{\prime}(s)=\cos \hat{\alpha}(s)+\cos \hat{\beta}(s)$ and it follows from this and (3.17) that if $s \in\left[s^{+}, \rho s_{1}\right]$, then $l^{\prime}(s) \geq \varepsilon^{2} /\left(50 \pi^{4}\right)$ if $s$ is good and $l^{\prime}(s) \geq-2$ even if $s$ is not good. We see from these estimates and Lemma 3.19 that, if $0<\varepsilon<10 \pi$,

$$
\begin{aligned}
\int_{s^{+}}^{\rho s_{1}} l^{\prime}(s) d s \geq & \frac{\varepsilon^{2}}{50 \pi^{4}} \text { length }\left\{s \in\left[s^{+}, \rho s_{1}\right]: s \text { is good }\right\} \\
& \quad-2 \text { length }\left\{s \in\left[s^{+}, \rho s_{1}\right]: s \text { is not good }\right\} \\
\geq & \frac{\varepsilon^{2}}{50 \pi^{4}}\left\{(\rho-2) s_{1}-s_{1}\right\}-2 s_{1} \\
> & 12 s_{1} \quad \text { by }(3.10) \\
> & 12 A r_{1} \quad \text { by }(3.14) \\
\geq & 12 a r_{1} .
\end{aligned}
$$

Since this contradicts (3.13), we must have $\pi-\hat{\alpha}\left(s^{+}\right)-\hat{\beta}\left(s^{+}\right) \leq \varepsilon /(5 \pi)$. A similar argument shows that $\pi-\alpha\left(s^{-}\right)-\beta\left(s^{-}\right) \leq \varepsilon /(5 \pi)$. Thus (3.15) is true, which completes the proof of the proposition. 
Proposition 3.13 implies that $U$ vanishes almost everywhere. It now follows in the same way as in the proof of Theorem 2.2 that the curvature vanishes everywhere.

\section{References}

[1] W. Ballmann, M. Brin \& K. Burns, On surfaces with no conjugate points, J. Differential Geometry 25 (1987) 249-273.

[2] K. Burns, The flat strip theorem fails for surfaces with no conjugate points, Preprint, 1990.

[3] C. B. Croke, Rigidity for simply connected manifolds with no conjugate points which are flat outside a compact set, Preprint, 1989.

[4] P. Eberlein \& B. O'Neill, Visibility manifolds, Pacific J. Math. 46 (1973) 45-109.

[5] J.-H. Eschenburg, Horospheres and the stable part of the geodesic flow, Math. Z. 153 (1977) 237-251.

[6] L. W. Green, Surfaces without conjugate points, Trans. Amer. Math. Soc. 76 (1954) 529-546.

[7] L. Green \& R. Gulliver, Planes without conjugate points, J. Differential Geometry 22 (1985) 43-47.

[8] E. Hopf, Closed surfaces without conjugate points, Proc. Nat. Acad. Sci. 34 (1948) 47-51.

[9] N. Innami, The n-plane with integral curvature zero and without conjugate points, Proc. Japan Acad. 62 (1986) 282-284.

[10] _ Euclidean metric and flat metric outside a compact set, Proc. Amer. Math. Soc. 105 (1989) 701-705.

[11] W. Klingenberg, Riemannian Geometry, Studies in Math. 1, de Gruyter, Berlin, 1982.

[12] J. J. O'Sullivan, Riemannian manifolds without focal points, J. Differential Geometry 11 (1976) 321-333.

NORTHWESTERN UNIVERSITY

UNIVERSITÄT GÖTTINGEN 